\title{
Effect of Carrier Transport on Thermal Distribution in Laser Diode
}

\author{
Dr. M. I. Azawe \\ Department of Physics / College of Education \\ University of Mosul
}

\section{Received}

$21 / 11 / 2007$
Accepted

03 / 03 / 2008

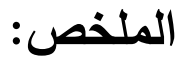

إنموذج تحليلي شامل لأجل نمذجة التأثيرات الح رارية الذاتية لثنائي الليزر ويعمل بتيار

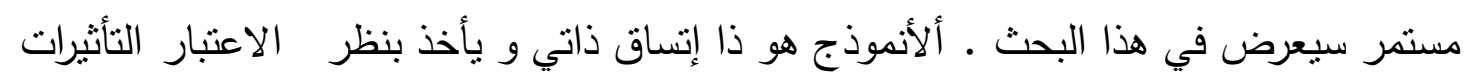

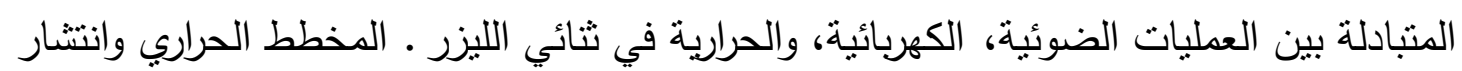

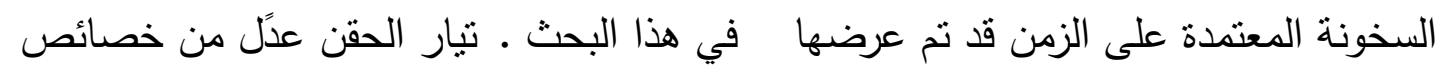

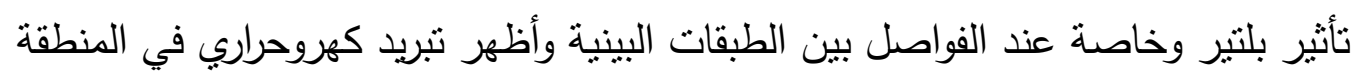

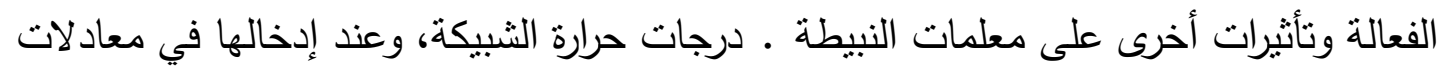
المعدل، لوحظ بأنها تزيح تردد النمط الرئيسي لفجو ة الليزر ـ مخطط حراري و بثلاثة أبعاد قد تم

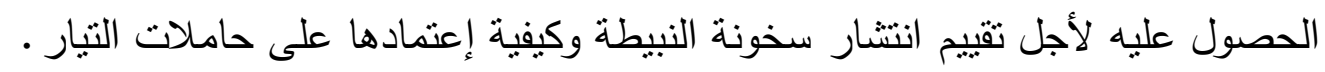

\section{ABSTRAC:}

A comprehensive numerical model to simulate self-heating effects of laser diode under continuous-wave operation is presented. The model is self-consistently accounts for the close interaction between optical, electrical, and thermal processes in the laser diode. Electro-thermal simulation offers the possibility of examining the effects on device performance of structure. Thermal profiles and time-dependent heat diffusion is presented in this investigation. Current injection modified the Peltier coefficient at interfaces; and gives rise to thermoelectric cooling at the active region and on the device parameters. Lattice temperatures, included in the rate equations were found to shift the frequency of the resonant dominant mode of the laser diode emission. 3D thermal plot of the thermal distribution was obtained in order to assess the heat diffusion as carrier-dependent for the laser diode.

\section{Introduction}


Stable emission of a semiconductor laser is very crucial in most applications of this laser [1], such as; optical communication, optical read-out systems (CD or DVD players, printers, scanners, etc.) and any other industrial or scientific application. Due to strong temperature dependencies of threshold current and quantum efficiency [2], and device lifetime[3], the laser diode (LD) mounting design must involve placing it on a Peltier thermoelectric cooler [4] or by fabrication a surface cooler [5]. The performance of Peltier device is mostly determined by its figure of merit [6]. Latest structure devices based on thermionic emission are expected to offer large thermoelectric power than bulk material due to the increase of Seebeck coefficient by the selective emission [7]. Heat management of the LD mount can be very critical issue and detrimental to device performance [8]. Hence, any theoretical model has to take into account the thermal dynamics of the LD [9]. Several sources of heating have identified and modeled the heat transport in LD device through thermal conduction $[10,11]$. The main effect of heating in LD device is the change in the background refractive index and hence a shift in the cavity resonance [12]. A hysteresis was observed in the LD absorption spectrum as result of thermal effects, due to the slow variation of the temperature of LD with injection current, with a thermal response time of milliseconds [13]. Nonlinear effects in heat conduction in the semiconductor exist, i.e., both thermal conductivity and diffusivity are temperature dependent [14]. These effects will be excluded here, but will be taken into consideration in the next article of the thermal dynamics of the LD.

This article presents a theoretical model to study the effects of thermal conduction in LD and their influences on the dynamics by the set rate equations and heat transfer mechanism. The model allows investigating the time-dependent solutions and spatial variation of temperature inside the laser cavity. The numerical simulations predict new phenomena exist in the LD cavity as will be shown in this article.

The article is organized as follows. Section II gives the theoretical model. The first part of the model deals with the inclusion of lattice temperature of the LD in single-mode rate equations to study its influence on the emission frequency. The second part is to establish the heat transfer equation from the active region to the surrounding layers and from the surrounding layers to the environment, in order to study the spatial temperature variation. Numerical simulations are presented in Section III. Section IV presents a summary and the conclusions of this investigation.

\section{Theoretical Model}




\section{Single-mode rate equations}

Laser cavity can be described by a set of rate equations, for $\xi$, the slowly varying complex amplitude of the optical field; $\stackrel{L}{\mathcal{N}}$ the normalized carrier density to transparency; and $\mathcal{J}$ is the lattice temperature normalized to the ambient temperature; are [1]:

$$
\begin{aligned}
& \frac{d}{d t} \xi=i \kappa \theta \xi+\kappa(1+i \alpha)(\Gamma G-1) \xi \\
& \frac{d}{d t} \mathcal{N}=-\gamma_{\mathrm{N}}[\mathcal{N}-\mathrm{J}+\mathrm{G} \mathcal{P}] \\
& \frac{d}{d t} \mathcal{T}=-\gamma_{\mathrm{T}}\left\{\mathcal{T}-1-\mathrm{Z} \mathcal{N}-\mathcal{J}^{2}\right\}
\end{aligned}
$$

In Eqn.(1), $\boldsymbol{\kappa}$ is the cavity decay rate, $\alpha$ is the linewidth enhancement factor, $\Gamma$ is the optical confinement factor, and $G$ is the nonlinear gain in the form [7]:

$$
\mathrm{G}(\mathcal{N}, \mathcal{P})=(\mathcal{N}-1) /(1+\epsilon \mathcal{P})
$$

Where

$\boldsymbol{\epsilon}$ is the self - saturation coefficient, and $\mathcal{P}$ is the photon number:

$$
\mathcal{P}(\mathrm{t})=|\xi(t)|^{2}
$$

$\gamma_{\mathrm{N}}$ in Eqn.(2) is the carrier decay rate, and J is the normalized carrier:

$$
\mathrm{J}=\frac{\mathrm{I}}{\gamma_{\mathrm{N}} \mathrm{e} \mathrm{N} \mathrm{V}_{1}}
$$

I is the bias current, $e$ is the electronic charge, $N_{\circ}$ is the carrier density at transparency, and $V_{1}$ is the volume of the active region.

Eqn.(3) represents the lattice temperature variations due to $\mathrm{ZN}$, the heating from nonradiative recombination, and $J^{2}$ heating from the Joule effect. $\mathrm{Z}$ is the coefficient due to nonradiative recombination thermal effect, and $\mathcal{J}$ is the Joule heating constant. $\gamma_{\mathrm{T}}$ is the temperature decay rate.

$\theta_{s}$ in the field equation is the detuning parameter from $\theta_{o}$ that varies linearly with temperature [11]:

$$
\theta=\theta_{o}-\epsilon_{T}(\mathcal{T}-1)
$$

$\epsilon_{T}$ is the coupling strength between the optical field and the temperature.

Suppose the solution of the rate equations are in the form:

$$
\xi(t)=E(t) e^{i \omega t}, \mathcal{N}(\mathrm{t})=\mathrm{N} \text {, and } \mathcal{T}(\mathrm{t})=\mathrm{T}
$$


So, after substituting in rate equations, we get the steady-state solution of Eqn.(1) in the form of, and with equating real and imaginary parts:

$$
\begin{aligned}
& \Gamma G=1 \\
& \omega=\boldsymbol{\kappa}(\theta+\alpha)
\end{aligned}
$$

Using Eqn.(4), at steady-state, we have:

$$
N=1+G=1+\frac{1}{\Gamma}
$$

The steady-state of Eqn.(2) is:

$$
\mathcal{P}(\mathrm{t})=|\mathrm{E}|^{2}=(\mathrm{J}-\mathrm{N}) \Gamma
$$

The steady-state solution of Eqn.(3) is:

$$
T=1+Z N+J^{2}
$$

Using Eqn.(4) and Eqn.(5), the carrier density can be written in the form:

$$
N=1+\left(1+\epsilon|E|^{2}\right) G
$$

The lasing threshold will be of the form:

$$
J_{t h}=1+G
$$

This gives us:

$$
|E|^{2}=\frac{\Gamma\left(J-J_{t h}\right)}{1+\epsilon_{T}}
$$

the photon number above threshold.

From Eqn.(10) and using Eqn.(7) and Eqn.(13), we get:

$$
\left.\omega=\boldsymbol{\kappa}(\theta+\alpha)=\boldsymbol{\kappa}\left\{\left[\theta_{o}-\epsilon(Z N+\mathcal{J}]^{2}\right)\right]+\alpha\right\}
$$

Or:

$$
\begin{aligned}
\omega-\omega_{\text {th }} & =-\boldsymbol{\kappa} \epsilon\left[Z\left(N-N_{t h}\right)+\mathcal{J}\left(J^{2}-\mathrm{J}_{\text {th }}{ }^{2}\right)\right]+\boldsymbol{\kappa} \alpha \\
& =-\boldsymbol{\kappa}\left[Z \epsilon(1+\epsilon)^{-1}\left(\mathrm{~J}-\mathrm{J}_{\text {th }}\right)+\mathcal{J}\left(J^{2}-\mathrm{J}_{\text {th }}{ }^{2}\right]+\boldsymbol{\kappa} \alpha\right.
\end{aligned}
$$

Eqn.(18) gives the emission frequency shift due to heating effects. This frequency shift, resulting from the nonradiative recombination and Joule heating, has to be included in any rate equations from now on. The unique feature of semiconductor laser is the lasing frequency dependent on the refractive index that varies with carrier density (pumping) [12].

\section{Heat transfer}


We have to identify the sources of heating in order to establish the heat transfer equations between the active layer, the surrounding layers, and the outside of the laser. We assume that the heat transfer obeys Newton's law of cooling. The main sources of heating are $[3,10]$ :

i- nonradiative recombination in the active layer,

ii- reabsorption of stimulated and spontaneous radiation,

iii- Joule heating.

The energy equation (heat conduction) will be developed and will be based on energy conservation for an elemental volume.

For active region:

Rate of change of heat (storage of energy) $=$

transport of heat from active region (net rate of flow)

+ rate of energy generated by the recombinations

$$
\begin{aligned}
\rho_{1} c_{1} \frac{d T_{1}}{d t} & =\frac{k_{1}}{V_{1}}\left(T_{2}-T_{1}\right) \\
& +\left(A_{n r} \exp \left\{\frac{E}{K}\left(\frac{1}{T_{a}}-\frac{1}{T_{1}}\right)+C N^{2} \exp \left(\frac{1}{T_{a}}-\frac{1}{T_{1}}\right)\right\}\right) N E_{g}
\end{aligned}
$$

For the surround:

Rate of change of heat of the surround

$=$ heat transfer

t heat generated from the absorption of the lasing mode photons ouside the active region

+ absorption of spotaneous emission that deviates outside the active region

$\rho_{2} c_{2} \frac{d T_{2}}{d t}=\left(\frac{k_{1}}{V_{2}}\left(T_{1}-T_{2}\right)+\frac{k_{2}}{V_{2}}\left(T_{a}-T_{2}\right)\right)+\frac{G|E|^{2}(1-\Gamma}{V_{2}} E_{g} V_{1}+$ $\frac{1-\Gamma \beta}{1+\gamma} \frac{B N^{2}}{V_{2}} E_{g} V_{1} \exp \left\{\frac{E}{K}\left(\frac{1}{T_{a}}-\frac{1}{T_{1}}\right)\right\}$

where $\rho_{i}, c_{i}, V_{i}, k_{i}$, and $T_{i}$ are the density, specific heat capacity, volume, heat transfer coefficient, and temperature respectively for the active region $(i=1)$ and surrounding layers $(i=2) . E_{g}$ is the dominant photon energy, $T_{a}$ is the ambient temperature, $K$ is Boltzmann's constant, $E$ is the activation energy, $A_{n r}$ is the nonradiative recombination constant, and $C$ is the Auger recombination constant. $\gamma$ is the ratio of spontaneous emission absorbed in the active region to the spontaneous emission absorbed in the surroundings, $\beta$ is the fraction of spontaneous emission coupled to the lasing mode, and $B$ is the radiative recombination constant. 
For isotropic medium, the thermal diffusivity $(D=k / \rho c)$ in $\mathrm{m}^{2} / \mathrm{sec}$ is constant and temperature independent [15].

The initial conditions of the above system are, at time $\mathrm{t}=0$ :

$$
N(0)=0, \mathcal{P}(0)=0, T_{1}(0)=T, \quad T_{2}(0)=T_{a}
$$

The above equations will be used for thermal profile and time-dependent heat diffusion.

For good understanding in the thermal mechanism of heat in semiconductor laser, two approaches have to be used. Macroscopic thermal diffusion due to convention at the laser's surface, and microscopic heat exchange processes that occur when carriers move across the heterojunctions. The model for thermal diffusion in multilayered semiconductor (isotropic) will be developed. Thermal diffusion equation can be written in a general form of Eqn.(19) as:

$$
\nabla^{2} T-\frac{1}{D} \frac{\partial T}{\partial t}+\frac{\mathbb{Q}}{k}=0
$$

where $\mathbb{Q}$ is the heat source, and as stated above (cf. Eqn.(19-20)) is both dependent on position and on time, i.e.,

$$
\mathbb{Q}=\mathbb{Q}(r, t)
$$

The thermal field evaluation of the semiconductor will be assumed to be as:

1- laser diode (sample)

2- laser mount

3- air surrounding.

This assumption allows us to write Eqn.(22) in the following form for the three media:

$$
\begin{aligned}
& \nabla^{2} T_{a}-\frac{1}{D_{a}} \frac{\partial T_{a}}{\partial t}=0 \\
& \nabla^{2} T_{s}-\frac{1}{D_{s}} \frac{\partial T_{s}}{\partial t}=-\frac{\mathbb{Q}(r, t)}{k_{s}} \\
& \nabla^{2} T_{m}-\frac{1}{D_{m}} \frac{\partial T_{m}}{\partial t}=0
\end{aligned}
$$

Where a stands for air, $\mathrm{s}$ for sample (semiconductor), and $\mathrm{m}$ for mount.

To complete the boundary value problem, boundary conditions and initial conditions must be specified. If two are in contact, temperature and heat flux continuity is imposed at contact boundaries (perfect thermal contact). Also, the dimensions extend to infinity (both the surface plane in (x,y)- direction as the air and mount in the perpendicular $\mathrm{z}$-direction). For this, we have the boundary conditions as:

$$
\begin{aligned}
& T_{a}(z=0)=T_{s}(z=0) \\
& T_{s}(z=-l)=T_{m}(z=-l)
\end{aligned}
$$

is the temperature continuity at the sample interfaces $(z=0$ and $z=-l)$. 


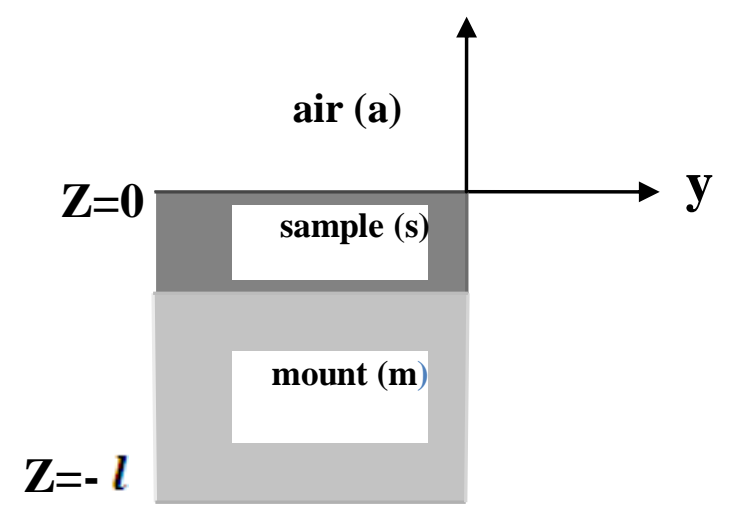

Fig. (1) Thermal field evaluation of the sample.

Also, the heat-flow continuity at the interfaces (see Fig.(1)):

$$
\begin{aligned}
& \left.k_{a} \frac{\partial T_{a}}{\partial z}\right|_{z=0}=\left.k_{s} \frac{\partial T_{s}}{\partial z}\right|_{z=0} \\
& \left.k_{s} \frac{\partial T_{s}}{\partial z}\right|_{z=-l}=\left.k_{m} \frac{\partial T_{m}}{\partial z}\right|_{z=-l}
\end{aligned}
$$

$\mathbb{Q}(r, t)$ can be expressed in cylindrical coordinates as[15]:

$$
\mathbb{Q}(r, t)=\left(\frac{P_{o} \alpha_{a b s}}{2 \pi a^{2}}\right) \exp \left(\alpha_{a b s} Z\right) \exp \left(\frac{-r^{2}}{a^{2}}\right) \exp (i \omega t)
$$

where $P_{o}$ is the absorbed power of the laser beam, $\alpha_{a b s}$ is the absorption coefficient, $a$ is the laser beam width, and $\omega$ is the angular frequency of the laser beam. To find an analytical expression for the temperature field distribution in the cylindrical geometry, a combination of Hankel and Laplace transformation will be used.

The Hankel transformation is [15]:

$$
\begin{aligned}
& T_{i}(r, z, t)=T_{0 j}(\delta, z, t) \\
& T_{o j}(\delta)=\int_{0}^{\infty} T_{i}(r) J_{o}(\delta r) r d r
\end{aligned}
$$

on the $\mathrm{i}-\mathrm{j}$ interface, and $J_{o}$ is the Bessel function of zero order. This transformation is applied to Eqn.(25) in order to reduce the partial differential equation to simpler partial differential equation.

$$
\begin{array}{r}
-\delta^{2} T_{o j}(\delta, z, t)+\frac{\partial T_{o j}}{\partial z^{2}}(\delta, z, t)-\frac{1}{D_{i}} \frac{\partial T_{o j}}{\partial t}(\delta, z, t)= \\
-\mathbb{Q}_{o j}^{I}(\delta) \exp \left(\alpha_{i} z\right) \delta(t)
\end{array}
$$

where $\mathbb{Q}_{o j}^{I}(\delta)$ is the Hankel transformation of $\mathbb{Q}_{i}^{I}(r)$. The Laplace transformation is: 


$$
\left.T_{o j}(\delta, z, t)=\right) \mathfrak{\Im}_{o j}(\delta, z, \rho)
$$

On Eqn.(34), we get:

$$
\begin{gathered}
-\delta^{2} \mathfrak{\Im}_{o j}(\delta, z, \rho)+\frac{\partial^{2} \mathfrak{\Im}_{o j}}{\partial z^{2}}(\delta, z, \rho)-\frac{\rho}{D_{i}} \mathfrak{I}_{o j}(\delta, z, \rho) \\
=-\mathbb{Q}_{o j}^{r}(\delta) \exp \left(\alpha_{i} z\right)
\end{gathered}
$$

The solution of Eqn.(36) in $\mathrm{z}$ as follows:

$$
\begin{aligned}
\mathfrak{\Im}_{o j}(\delta, z, \rho) & =A_{i}(\delta, \rho) \exp \left(-\beta_{i} z\right) \\
& +B_{i}(\delta, \rho) \exp \left(\beta_{i} z\right)+\frac{\mathbb{Q}_{o j}^{I}(\delta) \exp \left(\alpha_{i} z\right)}{\beta_{i}^{2}-\alpha_{i}^{2}}
\end{aligned}
$$

where $\beta_{i}^{2}=\delta^{2}+\left(i \omega^{t} D_{i}\right)$. Using the Hankel inversion as:

$$
T_{i}(r)=\int_{0}^{\infty} T_{o j}(\delta) J_{o}(\delta r) \delta d \delta
$$

The temperature field equation is obtained as:

$$
\begin{aligned}
\left.T_{i}(r, z, t)\right)= & \int_{0}^{\infty}\left[A_{i}(\delta) \exp \left(-\beta_{i} z\right)+B_{i}(\delta) \exp \left(\beta_{i} z\right)\right. \\
& \left.+\frac{\mathbb{Q}_{o j}^{r}(\delta)}{\beta_{i}^{2}-\alpha_{i}^{2}} \exp \left(\alpha_{i} z\right)\right] \exp (i \omega t) J_{o}(\delta r) \delta d \delta
\end{aligned}
$$

with $\mathrm{A}$ and $\mathrm{B}$ are constants.

A Matlab program will be developed to evaluate the integral in Eqn.(39) with the functions inside the integral will be computed in a Matlab program functions else. Eqn.(39) can be written for air, sample, and mount as shown in Fig.(1).

\section{Numerical Results}

This section represents the results of numerical simulations of the model developed after a reasonable choice of the laser parameters given in Table I. The other parameters, which are not stated in the table, will be described in the simulation as a variable.

The rate equations stated above [Eqn.(1)-(3)] will be first evaluated as an ordinary differential equations using the Matlab package with solutions based on an explicit Runge-Kutta fourth-order formula. The results show that the change of lattice temperature of the device with respect to the ambient temperature and its variation with carrier transport. As shown in Fig.(2), the temperature rise in the LD device diverged greatly after time around the carrier lifetime ( $3 \mathrm{nsec}$ ), which is the time enough for the population inversion to build-up. Also, as can be seen from the graph, the temperature distribution inside the device is not uniform. This behaviour will be illustrated in depth in the following arguments. Detuning in the lattice temperature of the device with the 
injection current had a very high thermal response very less than milliseconds (cf. Ref [13]).

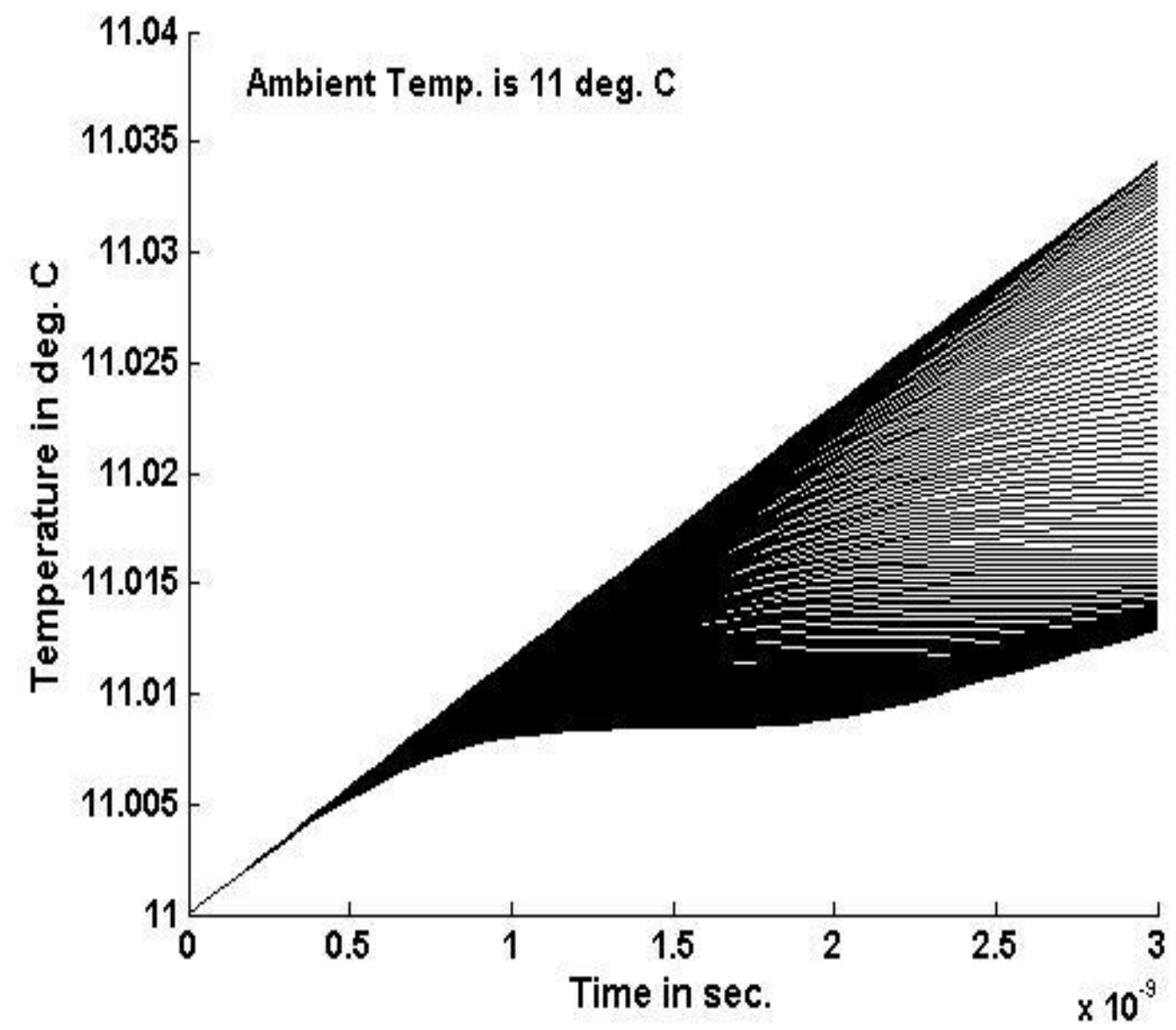

Fig.(2): The variation of lattice temperature of the $L D$ device with time.

The main effect of heating in the LD, as stated, is the change in the refractive index and hence a shift in the laser emission frequency, as given in Eqn.(18). The shift, due to the main thermal effects; Joule heating, nonradiative recombination, is shown in Fig.(3). This shift has to be included in any investigation of LD emission spectrum. The decrease in the emission frequency with injection current (carriers) is not linear due to the increase of these thermal effects with pumping current. As we know that the shift of the lasing mode with current, due to the bandfilling effect, has given a great attention [13]. This shift, due to bandfilling, and the shift due the thermal effect have to be studied closely and experimentally in order to get a better understanding of the frequency detuning of the lasing mode, provided that the temperature of the mount has to be perfectly stable. 


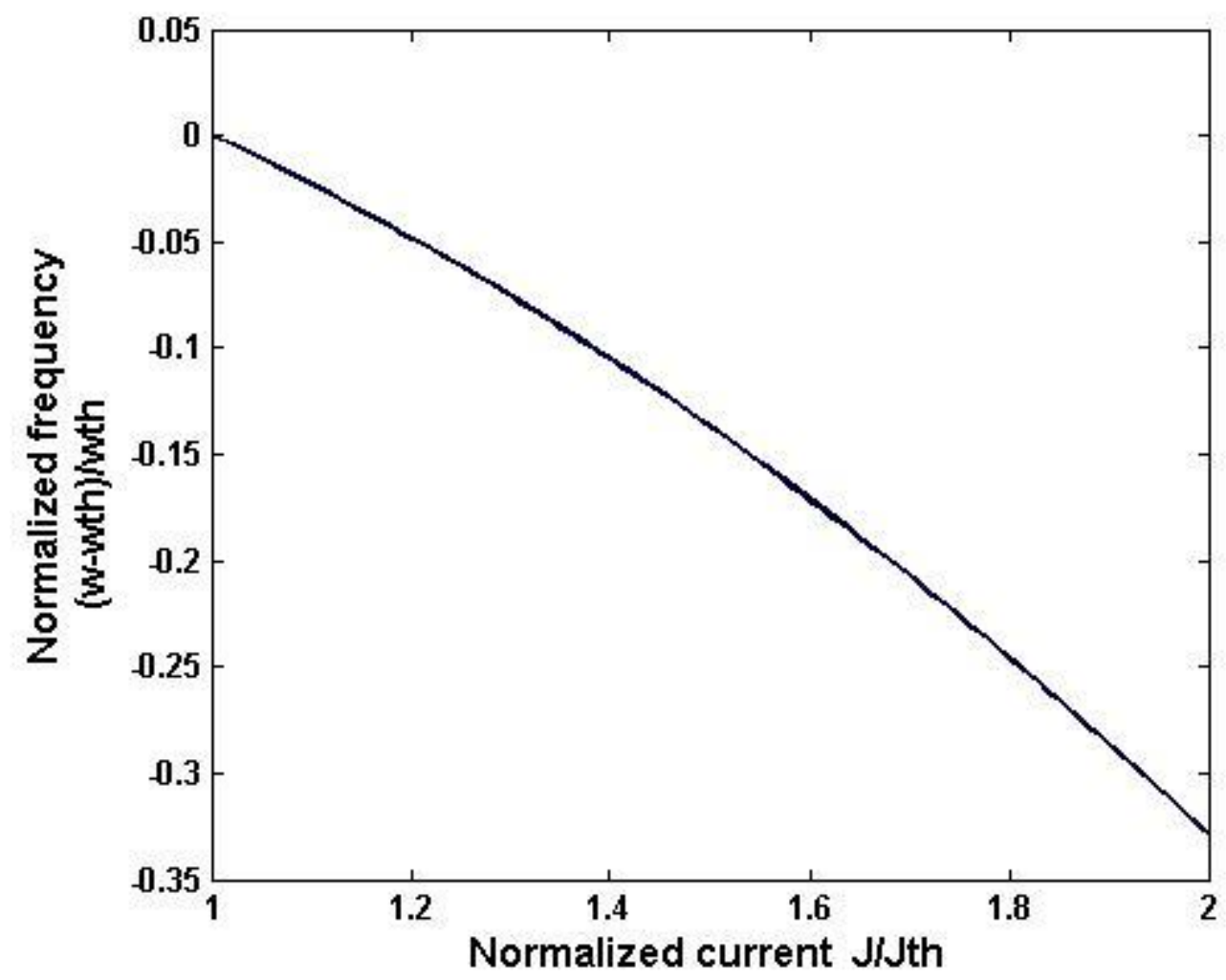

Fig.(3):Frequency shift of $L D$ resonance cavity mode with normalized injection current.

To study the heat transfer and its interplay between electrical and thermal effects, assuming uniform distribution of carriers in the active region, time-dependent and spatial variation will be investigated thoroughly.

Numerical solutions of Eqns.(19) \& (20) exhibit many time-scales plots. For short time-scale, around the carrier lifetime (in nsec.), i.e., when the electron concentration is varying, the active region temperature rises slowly and remain very close to the ambient temperature, as shown in Fig.(4). While the surrounding temperature rises sharply as a result of heat generation due to reabsorption of photons outside the active region. This figure illustrates the time-dependent of heat diffusion of the LD. These numerical solutions were obtained after establishing the boundary conditions and the current was taken to be switched on at time $t=0$, i.e., the photon and electrons densities are negligible in the active region and the device will be at ambient temperature. The solutions were performed in the Matlab (using the code ODE45) package. 


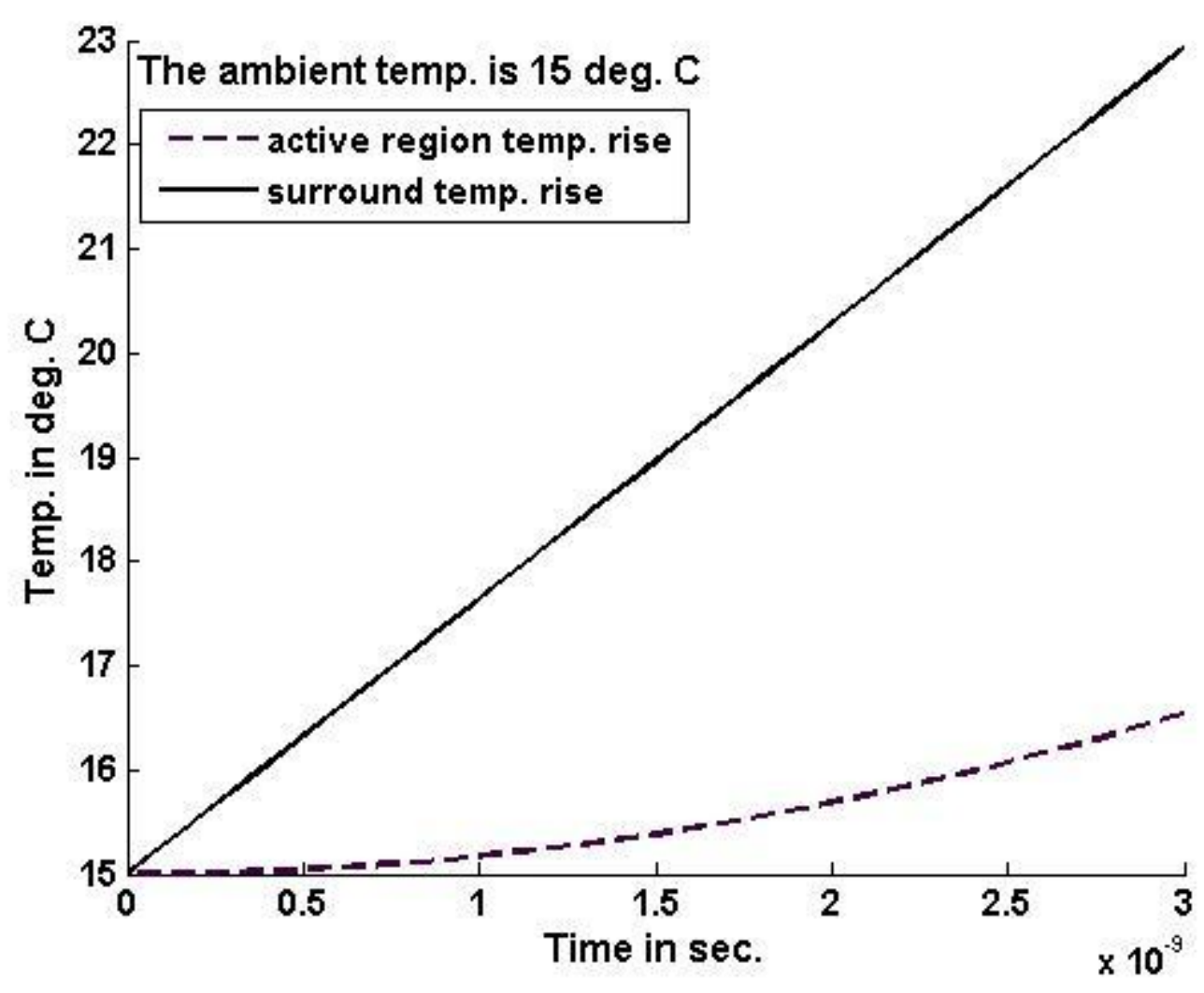

Fig.(4):Temperature rise of both the active region and the surround with time in the scale of carrier lifetime.

The ambient temperature was taken to be $15^{\circ} \mathrm{C}$.

For longer time-scale (greater than carrier lifetime), as shown in Fig.(5), the temperature variation of both the active region and the surround were plotted in logarithmic scale. An interesting feature of the thermal profile of LD is illustrated in the graph. The active region shows a thermoelectric cooling, while the surroundings show the associated thermoelectric heating and is mostly transferred to location near the Peltier cooler down to the heat sink. Thermal diffusivity occurs in LD device interfaces in a nonlinear manner. This behaviour has to be given some attention since it is very useful in decreasing the temperature of the LD device and will increase the quantum efficiency and device lifetime. 


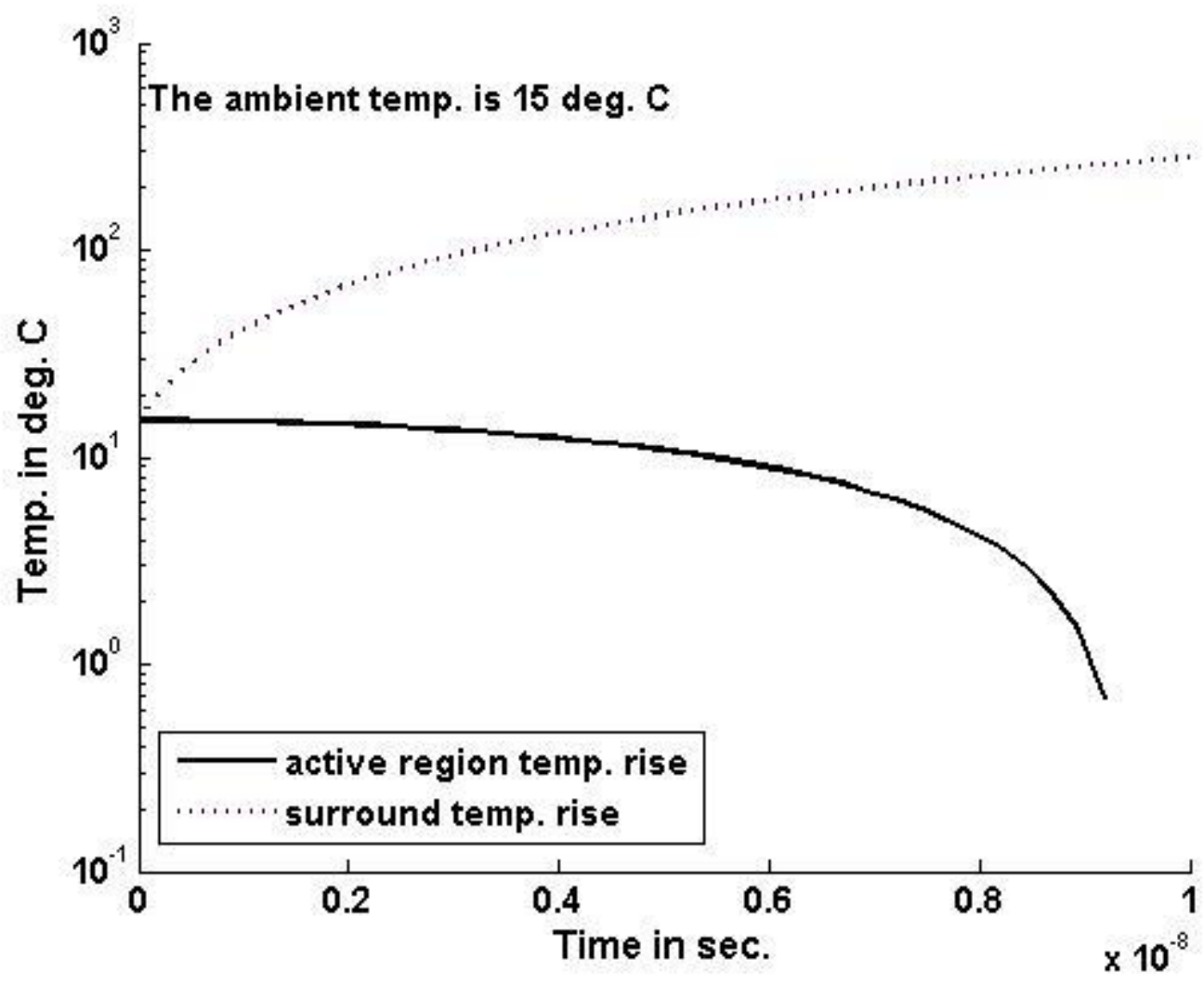

Fig.(5):Time-dependent of heat transfer of the LD device for both the active region and the surround.

To investigate the spatial distribution (3D) of heat transfer of the LD device, Eqn.(39) has to be evaluated for both the active region and the surround by Matlab (using the quad8). The Matlab source code is for calculating the integrals, but the functions inside the integral will be required by the Matlab syntax. The spatial distribution will be parallel and perpendicular to the active region.

The LD is assumed to be stripe geometry, which is the most popular device for many benefits, so the current spreading in the active region will be restricted to a small portion under the stripe. Hence, the heat diffusion as a result of the current transport in the device, the active region and the cladding layers has to be evaluated in order to get the whole thermoelectric distribution. 3D thermoelectric physical device simulation can be helpful, since the LD epitaxial layers are partitioned and electrically connected to each other. 


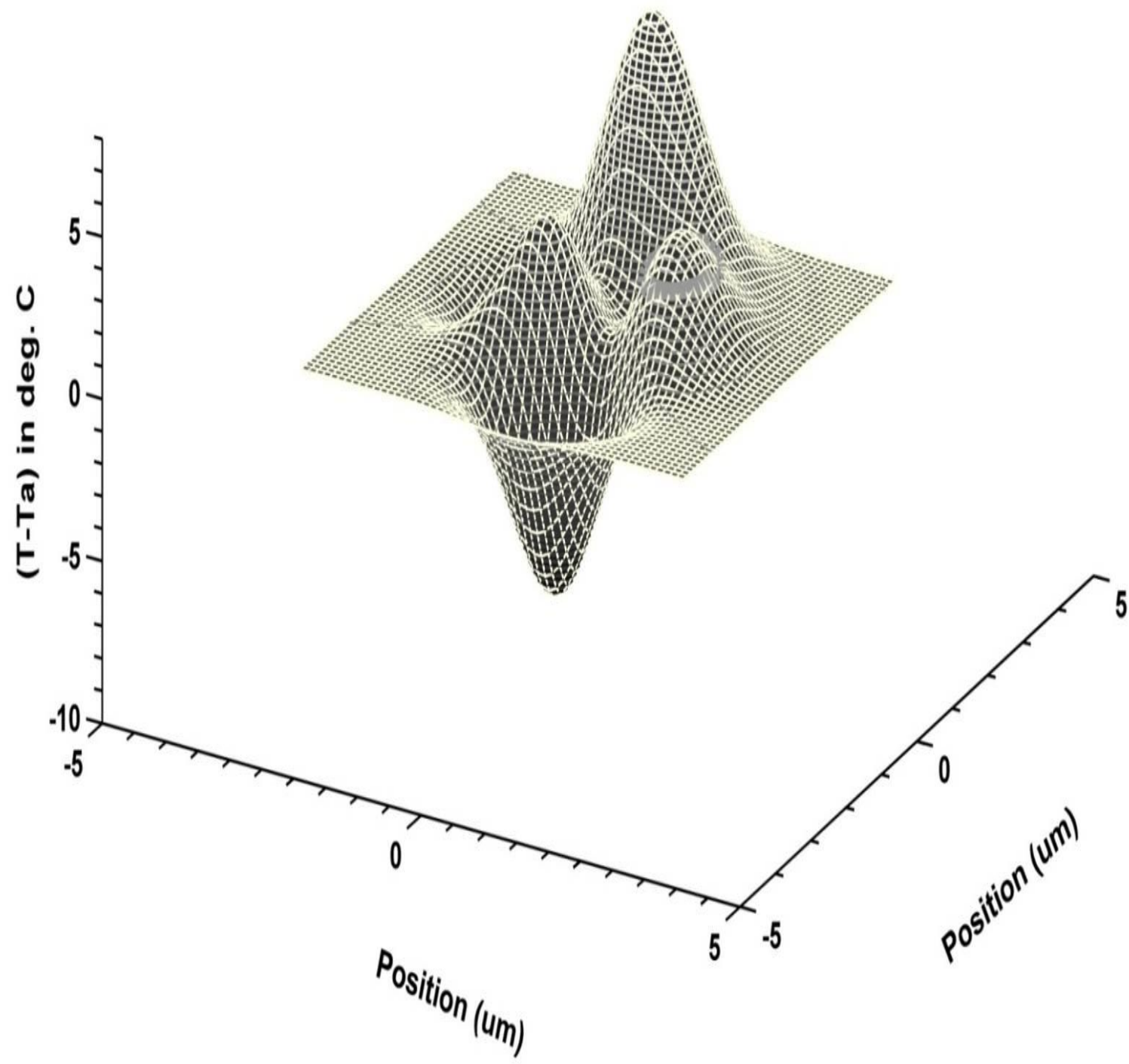

Fig. (6): Thermal distribution of the heat transfer of the LD parallel and perpendicular to the active region.

The behaviour of the 3D thermal distribution is shown in Fig.(6). As can be seen from the graph, a temperature dip around the active region was occurred due the electron-hole recombination radiative process inside the active region. An increase in the temperature of the surrounding regions was observed because of the reabsorption of the emitted photons and the nonradiative recombination process outside the active region. 


\section{Summary and Conclusions}

In summary, thermal characteristics of the LD device were investigated, and thermoelectric diagrams were obtained. Carrier transport perpendicular to the active region, sandwiched between two heterostructure cladding layers, thermoelectric cooling and heating were observed. A comprehensive numerical model to self-consistently simulate the coupled rate equations for electronic and optical interaction, and thermal process using the rate of heat transfer of the LD, were developed. Thermal diffusion equations were solved with proper boundary conditions for temperature distribution over the device. The hot carriers and nonequilibrium LO phonons were found to result in an additional carrier-temperature rise and more severe thermal rollover in the surroundings and hence lowering the lifetime of the LD and its quantum efficiency. Thermoelectric cooling (Peltier effect) in the active region was observed due to the hole capture by radiative process whence the carrier inversion started. Numerical simulations suggesting that the rise in the lattice temperature is very crucial and gives an effective frequency shift resonant mode of the laser cavity. Also, the numerical analysis of the heat transfer for 3D thermal distribution of the LD device shows a large temperature rise in the interface of the heterojunctions due to Auger heating effect and lattice mismatch that may exist. Proper design of the LD can eliminate the need for Peltier cooler when carrier transport can occur only by tunnelling, i.e., in case of quantum cascade laser, and with no nonradiative processes. 


\section{Table I \\ Laser Parameters}

\begin{tabular}{|c|c|c|c|}
\hline Symbol & Meaning & Typical value & Units \\
\hline$\alpha$ & Linewidth enhancement factor & 3 & - \\
\hline$\Gamma$ & Lasing mode confinement factor & 0.3 & - \\
\hline$\kappa$ & Cavity decay rate & 300 & $(\mathrm{nsec})^{-1}$ \\
\hline $\mathrm{d}$ & Active layer thickness & $0.1 \times 10^{-6}$ & $\mathrm{~m}$ \\
\hline $\mathrm{W}$ & Active layer width & $50 \times 10^{-6}$ & $\mathrm{~m}$ \\
\hline $\mathrm{L}$ & Cavity length & $300 \times 10^{-6}$ & $\mathrm{~m}$ \\
\hline$Y_{\mathrm{N}}$ & Carrier decay rate & .33 & $(\text { nsec })^{-1}$ \\
\hline$\gamma_{T}$ & Temperature decay rate & $5 \times 10^{-3}$ & $(\mathrm{nsec})^{-1}$ \\
\hline $\mathrm{Z}$ & Nonradiative recombination heating & 0.3 & - \\
\hline J & Joule heating & $5 \times 10^{-4}$ & - \\
\hline$\epsilon$ & Self-saturation coefficient & $1 \times 10^{-2}$ & - \\
\hline$\epsilon_{T}$ & Detuning thermal coefficient & 200 & - \\
\hline$A_{n r}$ & Nonradiative recombination constant & $10^{8}$ & $\sec ^{-1}$ \\
\hline$B$ & Band-to-band recombination constant & 0.26 & $\sec ^{-1}$ \\
\hline$C$ & Auger recombination constant & $4.4 \times 10^{-10}$ & $\sec ^{-1}$ \\
\hline$\beta$ & $\begin{array}{l}\text { Fraction of spontaneous emission coupled } \\
\text { to lasing mode }\end{array}$ & $10^{-4}$ & - \\
\hline$\rho_{1}$ & Density of active region & - & Kg.m-3 \\
\hline$c_{1}$ & Specific heat of active region & - & J.kg-1K-1 \\
\hline$\rho_{1} c_{1}$ & For active region & $9.26 \times 105$ & J.m-3K-1 \\
\hline$k_{1}$ & Thermal conductivity active region & $5.556 \times 10^{-12}$ & $\mathrm{~W} \cdot \mathrm{m}^{-1} \mathrm{~K}^{-1}$ \\
\hline$\rho_{2} c_{2}$ & For the surround & $4.63 \times 10^{-3}$ & $\mathrm{~J} . \mathrm{m}^{-3} \mathrm{~K}^{-1}$ \\
\hline$k_{2}$ & Thermal conductivity of the surround & $2.083 \times 10^{-11}$ & $\mathrm{~W} \cdot \mathrm{m}^{-1} \mathrm{~K}^{-1}$ \\
\hline$E_{g}$ & Bandgap energy & $1.27 \times 01^{-19}$ & $\mathrm{~J}$ \\
\hline
\end{tabular}




\section{References:}

1. Liu, Y., Ng, W., Choquette, K. D., and Hess, K., IEEE J. Quant. Electron. QE-41,15 (2005).

2. Pipe, K. P. and Ram, R. J., Int. Mechanical Engineering Congress and Exposition, New York, (2001).

3. Pipe, K. P., Ram, R. J., and Shakouri, A., IEEE Photon. Technol. Lett. 14, 453 (2002).

4. Zhang, X., Wen, J., and Sun, C., Appl. Phys. A-76,261(2003).

5. Fan, X. et al., Appl. Phys. Lett. 78, 1580 (2001).

6. Chao, K. A., Larsson, M., and Mal'shukov, A. G., Appl. Phys. Lett. 87, 22103 (2003).

7. Bian, Z., et al., Appl. Phys. Lett. 88, 12102 (2006).

8. O’Dwyer, M. F., Lewis, R. A., Zhang, C., and Humphrey, T. E., Phys. Rev. B-72,330 (2005).

9. Kozodoy, P., et al., IEEE Trans. Component \& Packing Technol. 28,651 (2005).

10. Smith, W. R., King, J. R., Tuck, B., and Orton, J. W., IMA J. Appl. Math. 63,1 (1999).

11. Masoller, C., Sorrentino, T., Chevrollier, M., and Oria, M., IEEE J. Quant. Electron. QE-43, 261 (2007).

12. Agrawal, G. P., and Dutta, N. K., Long Wavelength Semiconductor Lasers, Van Nostrand Reinhold, New York, 1986.

13. Palankovski, V., Schultheis, R., and Selberherr, S., IEEE Trans. Electron. Devices 48,1264 (2001).

14. Rinaldi,N., MACSI-net Workshop on "Optimization and Coupled Problems in Electromagnetism" (2001), e-mail: nirinald@unina.it

15. Bante, J., Murray, C., Dutchert, J., and Alvarado-Gil, J. J., Proc. SPIE 57761, 485(2005). 